\title{
Influence of the syringe model on the results of the International Dysphagia Diet Standardisation initiative flow test
}

Roberto Oliveira Dantas ${ }^{(1)}$ Luciana Oliveira ${ }^{(1)}$

Faculdade de Medicina de Ribeirão Preto da Universidade de São Paulo FMRRP/USP, Ribeirão Preto, São Paulo, Brasil.

Conflict of interests: Nonexistent

\section{(c) (i)}

Received on: February 7, 2018 Accept on: April 26, 2018

Mailing address:

Prof Dr. Roberto Oliveira Dantas Faculdade de Medicina de Ribeirão Preto Avenida Bandeirantes, 3900

14049-900 - Ribeirão Preto, São Paulo, Brasil

E-mail: rodantas@fmrp.usp.br

\section{ABSTRACT}

Objective: to investigate whether two different syringes yield different results in the International Dysphagia Diet Standardization Initiative (IDDSI) flow test to evaluate liquid consistency.

Methods: two 10-mL syringes (Bencton and Dickinson, manufactured in the United States, and Saldanha Rodrigues, manufactured in Brazil) were compared. Flow rate of water added with food thickener (maltodextrin, xanthan gum and potassium chloride) at three concentrations, and of barium sulfate at three concentrations was measured immediately after preparation and at 8 hours and 24 hours thereafter.

Results: flow rate of both water and barium sulfate was higher with the Bencton and Dickinson syringe, with discrepancies between the two syringes in the classification of fluid consistency according to the IDDI framework.

Conclusion: in the evaluation of the consistency of liquids by the IDDSI flow test, a Bencton and Dickinson syringe should be used, following the recommendations of the IDDSI group.

Keywords: Deglutition Disorders; Diet; Swallowing; Viscosity 


\section{INTRODUCTION}

Modification of food consistency is an important component of the treatment of dysphagia ${ }^{1}$. However, standard definitions for the nomenclature, viscosity and texture of different food consistencies have not been established and may vary considerably among hospitals and countries. Therefore, there is a need for international standardization of the terminology and precise definition of liquid and food textures, considering not only the performance of clinical tests but also the management of patients with dysphagia ${ }^{2}$.

To achieve this objective, the International Dysphagia Diet Standardization Initiative (IDDSI) developed standardized definitions (classified by numbers, names and colors) for texture-modified foods and liquids ${ }^{3}$. Classification of modified liquids consisted of four levels rated from zero (thin) to four (extremely thick) ${ }^{3,4}$. The method proposed for the development of this classification system was the IDDSI flow test, using a syringe. In this test, $10 \mathrm{~mL}$ of the test liquid is added to a $10-\mathrm{mL}$ syringe, and the volume remaining in the syringe after a 10 -second period of flowing is measured.

Nevertheless, it is difficult to assume that all $10-\mathrm{mL}$ syringes used in different centers across the countries are manufactured with the same configuration, which could affect the results obtained from the IDDSI flow test. This concern was considered by the IDDSI's board of directors. The hypothesis of this study is that the syringe configuration affects the results of the IDDSI flow test. We aimed to investigate whether two different syringes yield different results in this test. A correct classification of liquid consistencies is fundamental for the application of the recently validated IDDSI Functional Diet Scale ${ }^{5}$.

\section{METHODS}

This study did not involve human patients or experimental animals and was exempt from ethics committee approval.

Two 10-mL syringes were compared - Becton and Dickinson ${ }^{\circledR}(\mathrm{BD})$ (Franklin Lakes, NJ, USA) and Saldanha Rodrigues ${ }^{\circledR}$ (SR) (Manaus, AM, Brazil). BD syringe has a $61 \mathrm{~mm}$ length (from " 0 " to " $10 \mathrm{~mL}$ " marks), an outer diameter of $16 \mathrm{~mm}$, an internal diameter of 14 $\mathrm{mm}$, and syringe hub of $10 \mathrm{~mm}$. SR syringe has a 50 $\mathrm{mm}$ length (from "0" to " $10 \mathrm{~mL}$ " marks), outer diameter of $19 \mathrm{~mm}$, internal diameter of $18 \mathrm{~mm}$, and a syringe hub of $12 \mathrm{~mm}$.

The test was performed with water added with food thickener (maltodextrin, xanthan gum, and potassium chloride) at different concentrations $-1.2 \mathrm{~g}, 2.4 \mathrm{~g}$ and $3.6 \mathrm{~g}$ in $100 \mathrm{~mL}$ of water, barium sulfate at $1 \mathrm{~g} / 3 \mathrm{~mL}$ and $1 \mathrm{~g} / 1 \mathrm{~mL}$, and $100 \mathrm{~mL}$ of barium sulfate $1 \mathrm{~g} / 1 \mathrm{~mL}$ with $1.2 \mathrm{~g}$ of the thickener, all at room temperature.

The test consisted in adding $10 \mathrm{~mL}$ of the liquid test into the syringe (at vertical position) with the nozzle covered with the finger. The nozzle is released for 10 seconds, and the volume remaining in the syringe is measured after this period ${ }^{4}$. This procedure was repeated 10 times immediately after each texturemodified liquid was prepared (time zero), and also performed 8 hours and 24 hours thereafter.

Results of both syringes obtained at time zero were compared by the Mann-Whitney test, a non-parametric test that enables the comparison of two independent groups, without assuming normality of data distributions ${ }^{6}$. Statistical analysis was performed using the SAS software, version 9.2.

\section{RESULTS}

The volume remaining of water (Table 1) and thickened barium sulfate (Table 2) was lower in BD than in SR syringe after the 10-second period of flowing. This indicates that the flow rate $(\mathrm{mL} / \mathrm{s})$ of both fluids through the BD syringe was higher than through the SR syringe (Figures 1 and 2).

Classification of the consistency of water added with 3.6g of thickener differed between the syringes (IDDSI level 3 and level 4 for BD and SR, respectively) as well as for barium sulfate at $1 \mathrm{~g} / 3 \mathrm{~mL}$ (level 1 for $B D$ and level 2 for SR) and at $1 \mathrm{~g} / 1 \mathrm{~mL}$ (level 2 for BD and level 3 for $\mathrm{SR})$. No difference was found between the flow rates through the syringes measured at 8 hours and at 24 hours after the liquid preparation (Figures 1 and 2), and the differences in the classification of fluid consistencies according to the IDDSI framework between the two syringes were maintained. 
Table 1. Comparison between a 10-mL Bencton \& Dickinson (BD) and a 10-mL Saldanha Rodrigues (SR) syringe in the volume of thickened liquid remaining in the syringe after 10 seconds of flowing

\begin{tabular}{ccccccc}
\hline \multicolumn{7}{c}{$\mathbf{c}$ Thickener } \\
\hline & MEAN (SD) & MEDIAN & MEAN (SD) & MEDIAN & MEAN (SD) & M.6g \\
\hline BD & $4.7(0.2)$ & 4.8 & $9.5(0.3)$ & 9.4 & $9.9(0.1)$ & 9.8 \\
SR & $6.8(0.2)$ & 6.8 & $9.9(0.1)$ & 9.8 & $10.0(0.0)$ & 10.0 \\
p & $<0.01$ & & 0.01 & & $<0.01$ & \\
\hline
\end{tabular}

SD: standard deviation; Man-Whitney test

Table 2. Comparison between a 10-mL Bencton \& Dickinson (BD) and a 10-mL Saldanha Rodrigues (SR) syringe in the volume of barium sulfate remaining in the syringe after 10 seconds of flowing

\begin{tabular}{|c|c|c|c|c|c|c|}
\hline \multicolumn{7}{|c|}{ Barium sulfate (concentrations) } \\
\hline & \multicolumn{2}{|c|}{$1 \mathrm{~g} / 3 \mathrm{~mL}$} & \multicolumn{2}{|c|}{$1 \mathrm{~g} / 1 \mathrm{~mL}$} & \multicolumn{2}{|c|}{$1 \mathrm{~g} / 1 \mathrm{~mL}+1.2 \mathrm{~g}$ thickener } \\
\hline & MEAN (SD) & MEDIAN & MEAN (SD) & MEDIAN & MEAN (SD) & MEDIAN \\
\hline $\mathrm{BD}$ & $1.4(0.1)$ & 1.4 & $7.9(0.2)$ & 7.8 & $8.3(0.1)$ & 8.4 \\
\hline SR & $4.3(0.3)$ & 4.2 & $9.4(0.2)$ & 9.4 & $9.6(0.2)$ & 9.7 \\
\hline$p$ & $<0.01$ & & $<0.01$ & & $<0.01$ & \\
\hline
\end{tabular}

SD: standard deviation; Man-Whitney test

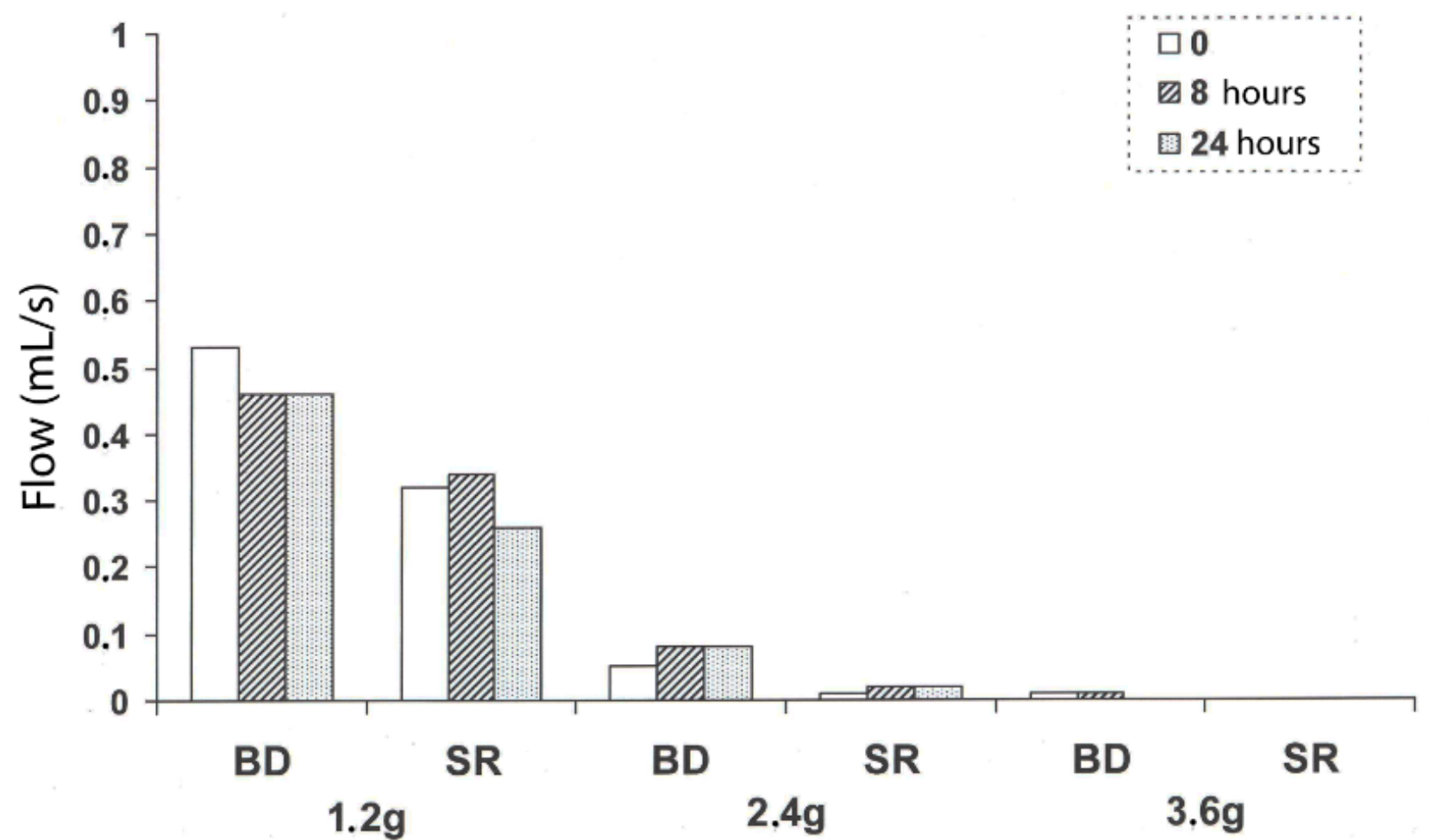

Legend: $\mathrm{BD}=$ Becton and Dickinson; $\mathrm{SR}=$ Saldanha Rodrigues

Figure 1. Flow $(\mathrm{mL} / \mathrm{s})$ through the Bencton \& Dickinson and Saldanha Rodrigues (SR) syringes of water $(100 \mathrm{~mL})$ added with food thickener at $1.2 \mathrm{~g}, 2.4 \mathrm{~g}$ and $3.6 \mathrm{~g}$ measured immediately after preparation (time 0 ), and at 8 hours and 24 hours after preparation. Flow rate was significantly higher in the Bencton \& Dickinson syringe $(p<0.01)$. 


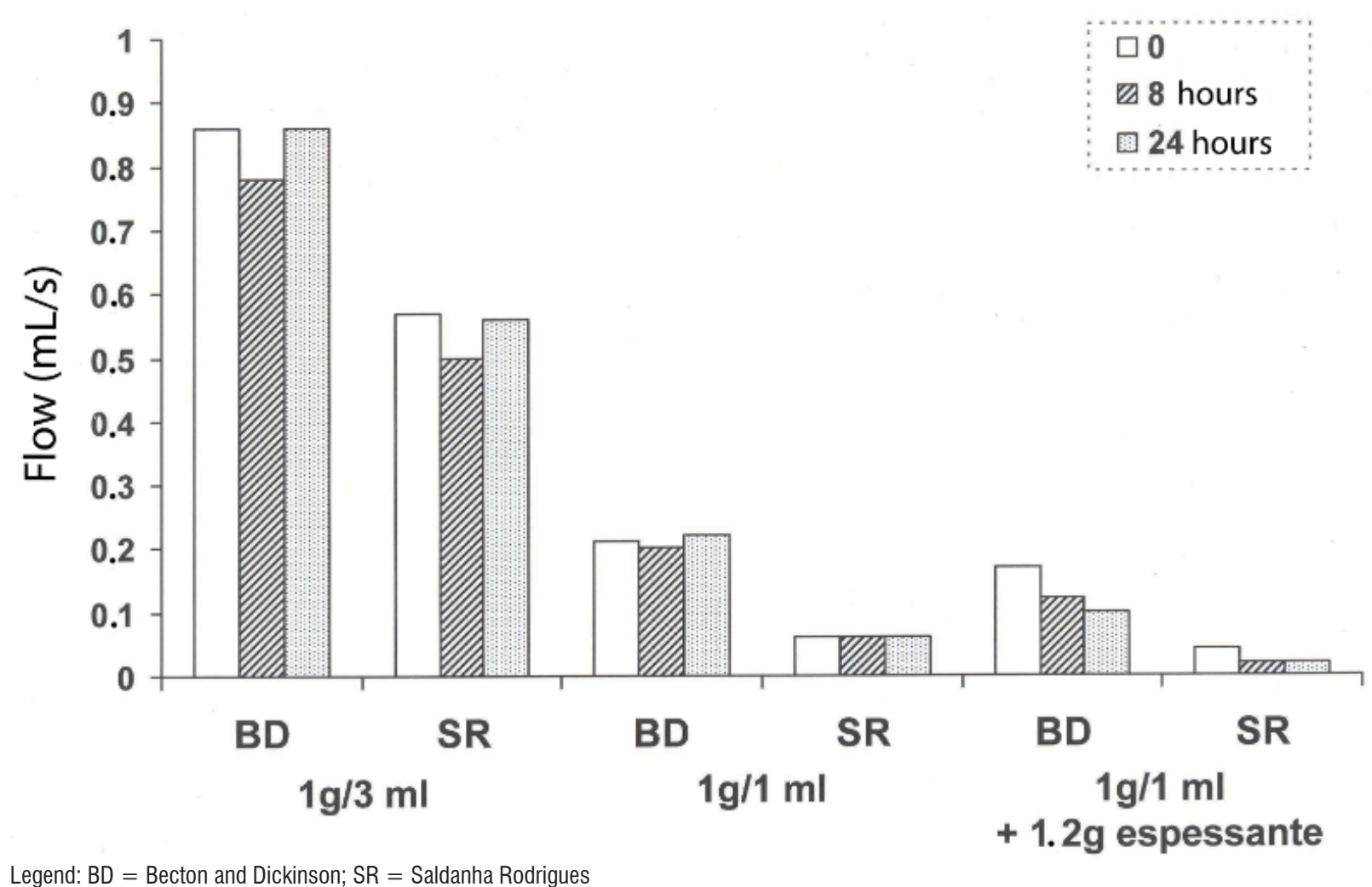

Figure 2. Flow rate $(\mathrm{mL} / \mathrm{s})$ through the Bencton \& Dickinson and Saldanha Rodrigues syringes of barium sulfate $(100 \mathrm{~mL})$ diluted with water at $1 \mathrm{~g} / 3 \mathrm{ml}, 1 \mathrm{~g} / 1 \mathrm{ml}$ and $1 \mathrm{~g} / 1 \mathrm{ml}$ added with $1.2 \mathrm{~g} \mathrm{f}$ food thickener measured immediately after preparation (time 0 ), and at 8 hours and 24 hours after preparation. Flow rate was significantly higher in the Bencton \& Dickinson syringe $(p<0.01)$.

\section{DISCUSSION}

The use of texture-modified liquid foods is of paramount importance in the treatment of dysphagia to avoid food aspiration in patients with oral feeding ${ }^{7,8}$. Hydration depends on fluid intake, and many patients are not able to swallow low-viscosity fluids, which are associated with a higher risk of aspiration ${ }^{8}$. Foods with high consistency are hence required for maintenance of a favorable nutritional status.

So far, institutions where dysphagia patients are diagnosed and treated have developed different understandings and terminologies for the terms "thin liquid", "paste", "solid", "thickened liquid", "pudding" and "nectar/honey-thick" liquids, "soft solid" consistency, "puree", among others, variable according to the country, the culture and eating habits of the population. Even though eating habits are influenced by cultural, social, ethnical and racial aspects that should be considered, especially in patients with digestive symptoms ${ }^{9}$; standardized characteristics and nomenclatures of food consistencies should be used across the countries.
The IDDSI was created with the aim to develop standardized terminology and definitions for texturemodified foods commonly used in the treatment of patients with dysphagia ${ }^{3}$. Categorization of consistencies by names, colors and numbers aims to facilitate the selection of the best food consistency for each patient. Standardized terminologies could be used in any place, irrespectively of the foods available and eating habits of each population, since the definitions proposed are for food consistencies, and not for foods themselves, which should be selected by those involved in patients' nutrition and hydration care.

For this reason, characterization of each consistency should be precise, so that a same consistency can be used in different hospitals and populations with varied cultures, languages, and food availability. This would be achieved by using a simple, practical, reproducible evaluation method. There is a close relationship between the degree of dysphagia and IDDSI framework, an important implication for diet therapy in dysphagia patients ${ }^{10}$.

For classification of fluids, the method proposed is the IDDSI flow test ${ }^{3,4}$, available at www.iddsi.org 
and already translated into Portuguese. The test was described with the use of a BD syringe, and the use of another syringe, with different manufacturing features, may yield different results, which was confirmed by our findings. In addition, syringes manufactured in different countries could also have distinct characteristics that, although may not compromise their usefulness for administration of injectable drugs, may result in different performance and outcomes in the IDDSI flow test. Thus, the test performed with syringes of different brands than BD do not produce the same results than those described in the IDSSI framework for liquid consistency definitions ${ }^{3,4}$.

The reason for different syringes produce different results may be associated with the Hagen-Poiseuille law: $Q=P \pi R^{4} / 8 L \eta$, where $Q$ is the volumetric flow rate, $P$ is the pressure, $R$ is the syringe radius, $L$ is the length, and $\eta$ is the viscosity coefficient of the fluid. Test with $B D$ and $S R$ syringes have the same $P$ and $\eta$, and the lower flow rate shown by the SR syringe may be associated with the length of the syringe tip. This was confirmed by the fact that, if only the syringe barrel were considered, it would be expected a lower flow rate with the $\mathrm{BD}$ syringe; however, the radius $(\mathrm{R})$ of the syringe hub is considerably reduced in relation to the barrel in both syringes and therefore the longer length of the tip was decisive for the lower flow rate.

Although their higher complexity and possible higher costs, other methods based on the same measuring principle of flow rate have been developed and should be tested ${ }^{11,12}$. The purpose of the IDDSI flow test is to provide a simple and fast way to determine the consistency of liquids and semi-liquids, without the use of expensive and highly complex equipment ${ }^{10}$. In this study, we did not evaluate the performance of the syringes in their primary purpose, i.e., administration of injectable drugs. Also, similar to previous results ${ }^{13}$, there was no significant changes in the consistencies of the texture-modified liquids within 24 hours of preparation, indicating their good stability.

\section{CONCLUSION}

In conclusion, the syringe selected for use in the IDDSI flow test for evaluation of fluid consistency affects its results, and therefore, a BD syringe should be used in the test, as recommended by the IDDSI group.

\section{REFERENCES}

1. Steele CM, Alsanei WA, Ayanikalath S, Barbon CEA, Chen J, Cichero JAY et al. The influence of food texture and liquid consistency modification on swallowing physiology and function: a systematic review. Dysphagia. 2015;30(1):2-26.

2. Cichero JAY, Steele CM, Duivestein J, Clavé P, Chen J, Kayashita J et al. The need for international terminology and definitions for texture-modified foods and thickened liquids used in dysphagia management: foundations of a global initiative. Curr Phys Med Rehabil Rep. 2013;1 (4):280-91.

3. Cichero JAY, Lam P, Steele CM, Hanson B, Chen $J$, Dantas RO et al. Development of international terminology and definitions for texture-modified foods and thickened fluids used in dysphagia management: the IDDSI framework. Dysphagia. 2017;32(2):293-314.

4. Hanson B. A review of diet standardization and bolus rheology in the management of dysphagia. Curr Opin Otolaryngol Head Neck Surg. 2016;24(3):183-90.

5. Steele CM, Namasivayam-McDonald AM, Guida BT, Cichero JA, Fuivestein J, Hanson B et al. Creation and initial validation of the International Dysphagia Diet Standardisation Initiative functional diet scale. Arch Phys Med Rehabil. 2018; (in press).

6. Conover WJ. Practical nonparametric statistic, $2^{\text {nd }}$ ed, New York: Wiley; 1980.

7. Vilardell N, Rofes L, Arreola V, Speyer R, Clavé P. A comparative study between modified starch and xantan gum thickeners in post-stroke oropharyngeal dysphagia. Dysphagia. 2016;31(2):169-79.

8. Newman R, Vilardell N, Clavé P, Speyer R. Effect of bolus viscosity on the safety and efficacy of swallowing and the kinematics of the swallowing response in patients with oropharyngeal dysphagia: white paper by the European Society for Swallowing Disorders (ESSD). Dysphagia. 2016;31(2):232-49.

9. Francisconi CF, Sperber AD, Fang X, Fukudo $S$, Gerson MJ, Kang JY et al. Multicultural aspects in functional gastrointestinal disorders (FGIDs). Gastroenterology. 2016;150(6):1344-54.

10. Su M, Zheng G, Chen Y, Xie H, Han W, Yang Q et al. Clinical Implication of IDDSI framework for texture recommendation for dysphagia patients. J Texture Stud. 2018;49(1):2-10.

11. Park NA, Cho Yl, Irvine TF. Steady shear viscosity measurements of viscoelastic fluids with the falling 
needle viscometer. J Non-Newtonian Fluid Mech. 1990;34(3):351-7.

12. Watanabe E, Yamagata $Y$, Kogirima M, Miyamoto K, Kayashita J. Development of a simple and objective evaluation method for thickened liquids using funnels. J Texture Stud. 2017;48(3):198-204.

13. Alves DC, Alves NA, Dantas RO. Consistency stability of water thickened with maltodextrin, xanthan gum and potassium chloride. $\mathrm{J}$ Texture Stud. 2017;48(6):530-3. 